\title{
Präoperative Opiate verstärken postoperative Schmerzen
}

\section{Opiate lindern Schmerzen - verur- sachen aber auch welche. Die schon länger diskutierte Annahme, dass eine präoperative Opioidtherapie postoperative Schmerzen verstärkt, wurde nun in einer Studie bei ortho- pädischen Patienten bestätigt.}

O pioide wirken nicht nur antinozizeptiv, sondern auch und zudem länger anhaltend pronozizeptiv, erläuterte Professor Arne May, Hamburg. Man vermutet deshalb seit einiger Zeit, dass präoperativ verabreichte Opioide kontraproduktiv sind, gegebenenfalls sogar Schmerzen nach einer Operation verschlimmern. Zu recht, wie eine aktuelle prospektive Beobachtungsstudie zeigte [Hina $\mathrm{N}$ et al. Eur J Anaesthesiol 2015; 32: 255-61]. Aufgenommen wurden 68 Patienten mit Osteoarthritis, die sich einer orthopädischen Operation unterziehen mussten und keine OpiatLangzeittherapie erhalten hatten. Ein Teil der Patienten $(n=28)$ erhielt wegen starker Schmerzen präoperativ $42 \mathrm{mg}$ Morphin-Äquivalent - und entwickelte vor der Operation eine Hyperalgesie. Laut May deutet das auf eine generali-

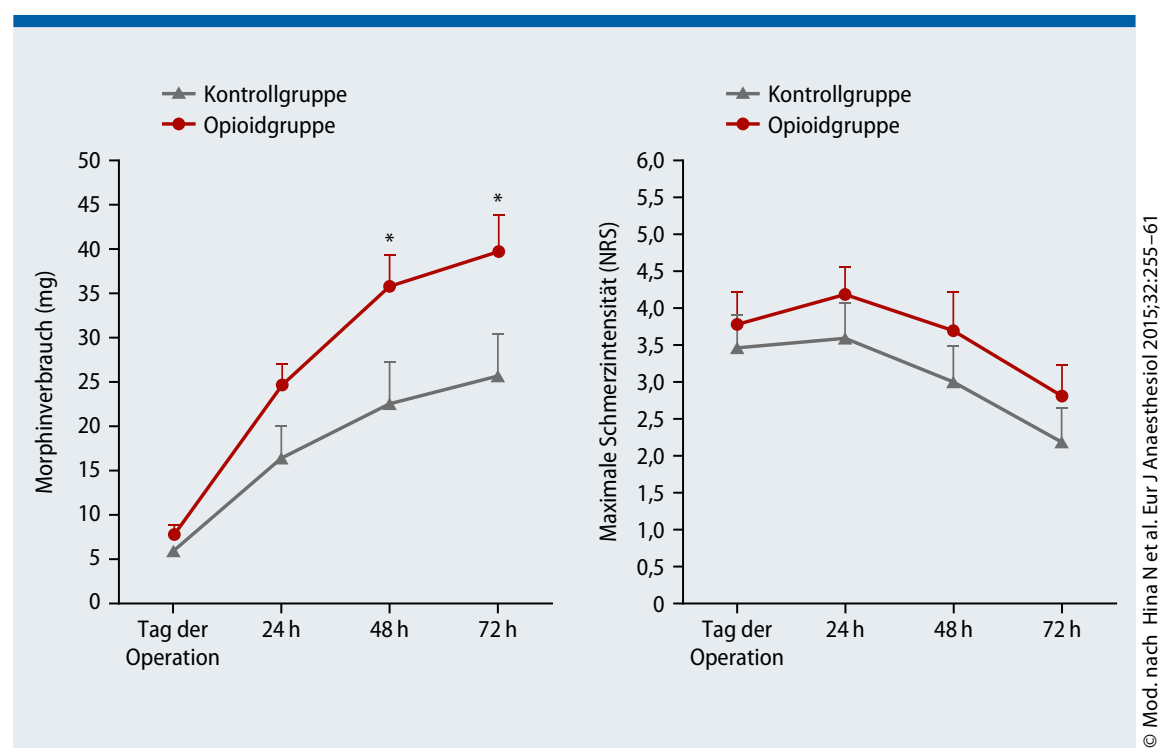

Abb. 1: OP-bedingte Schmerzen und Schmerzmittelverbrauch nach präoperativer OpioidGabe. Offenbar kann Opioideinnahme vor einer OP tatsächlich zu höheren postoperativen Schmerzen und höherem Verbrauch an weiteren Schmerzmitteln führen.

sierte Sensitisierung eventuell durch die niedrig-dosierte Opioideinnahme hin. Damit nicht genug, klagten sie im Aufwachraum signifikant häufiger über OPbedingte Schmerzen und benötigten mehr Schmerzmittel innerhalb der ers- ten 24 Stunden nach der OP als die Opioid-naiven Patienten (Abb. 1).

Ute Ayazpoor

Schmerz/Kopfschmerz (Arne May), 8. Neurologie-Update-Seminar, Mainz, 19.-20.2.2016

\section{Camptocormie: Wann hilft eine tiefe Hirnstimulation?}

Die Pathogenese der Camptocormie ist ungeklärt, ihre Therapie eine besondere Herausforderung. In einer aktuellen Studie war die tiefe Hirnstimulation (THS) erfolgreich, wenn sie spätestens ein Jahr nach Auftreten der Anteflexion durchgeführt wurde.

B ei der Camptocormie handelt es sich um eine starke Anteflexion der thorakolumbalen Wirbelsäule mit oder ohne Seitbeugung. Ihre Ursache ist laut Professor Jörg B. Schulz aus Aachen bis heute nicht geklärt. Diskutiert werden eine Dystonie der axialen Muskulatur, aber auch - und das halte er angesichts der überzeugenderen Berichte für wahrscheinlicher - eine Myopathie in der trunkalen Muskulatur. Ob die Myopa- thie aber eine Manifestation der Erkrankung oder eine Folge der medikamentösen Therapie ist, liegt noch im Dunklen, so Schulz.

Dem therapeutischen Nutzen einer THS ging eine aktuelle Untersuchung bei 25 Patienten mit idiopathischem Parkinson-Syndrom und zusätzlicher Camptocormie nach [Schulz-Schaeffer WJ et al. Mov Disord 2015; 14: 693 - 701]. Während bei 12 Patienten nach bilateraler Stimula- tion des Nucleus subthalamicus keine Veränderung der Camptocormie beobachtet wurde, verbesserte sich der Beugewinkel bei 13 Patienten um mindestens $50 \%$. Der Therapieerfolg war abhängig von der Dauer der Anteflexion. Das heißt, alle Patienten die maximal ein Jahr an Camptocormie litten, profitierten. Wechselhafte Ergebnisse wurden bei einer Symptomdauer zwischen 1,5 und 3 Jahren erzielt, bei Patienten mit Camptocormie seit mehr als 40 Monaten kam es zu keiner Verbesserung, berichtete Schulz. Die Autoren folgern daraus, dass die THS wohl bei voll ausgeprägter Muskelpathologie keinen Effekt mehr zeigt.

Bei der Entscheidung für eine THS sollten laut Schulz neben der Wirksamkeit mögliche Langzeitfolgen bedacht werden. Diskussionsstoff dazu lieferte eine gleichzeititg erschienene Untersu- 